\title{
Accelerative Effect of Olive Oil on Adrenal Corticosterone Secretion in Rats Loaded with Single or Repetitive Immersion-Restraint Stress
}

\author{
Hisanao TAKeuchI, ${ }^{*}$ Yutaka Kondo, Miki YanAgi and Masaki YoshiKAWA \\ Department of Applied Biological Chemistry, Faculty of Agriculture, Shizuoka University, \\ Shizuoka 422-8529, Japan
}

(Received July 22, 1999)

\begin{abstract}
Summary The present study was performed to clarify the effects of dietary oils on physiological and metabolic changes induced by a stress, using one-time or repetitive water-immersion of restrained rats (single or repetitive stress) as an experimental stress load. In rats fed any test diets containing $20 \%$ of the mixture of tripalmitin, tristearin, and corn oil (PSC), olive oil (OLI), safflower oil (SAF), and linseed oil (LIS) with repetitive stress loading, body weight gains and food intakes were generally reduced. The weights of the thymus and spleen also declined, but the adrenal weights were enhanced. Particularly, the increase in the adrenal weight of rats given the OLI diet was greater than of rats supplied with other diets. When the rats were loaded with the single or repetitive stress, the concentrations of urea, lipid peroxide, and corticosterone in the plasma were increased in rats fed any of dietary oils. The rise of plasma corticosterone level was especially great in rats fed the OLI diet. The concentrations of total cholesterol (T-CHOL) and triglyceride (TG) in the plasma and liver generally tended to be higher in rats fed the OLI diet than in rats given the other diets with and without stress exposure. Plasma corticosterone concentration was correlated to the adrenal weight $(r=0.87, p<0.05)$. This study showed that OLI especially enhanced the adrenal weight in rats exposed to the repetitive stress and further raised the increased secretion of adrenal corticosterone in rats loaded with the single or repetitive stress compared with the other oils. The mechanism explaining these actions of OLI was inferred to be related to the levels of T-CHOL and TG in the plasma and liver generally enhanced by stress.
\end{abstract}

Key Words olive oil, adrenal corticosterone, water immersion-restraint stress, single and repetitive stresses, total cholesterol

The appearance of physiological and metabolic changes (1-9) showing a decrease in body weight, a shrink of the spleen or thymus, an increase of adrenal weight, and so on $(10,11)$ are reported as the responses of a living body on acute (single) or chronic (repetitive) stress (5). A stress load to rats enhances the secretion of corticosterone, and this hormone is used as an index of stress in many studies $(4,5,7)$. An incorporation of cholesterol (CHOL) into the adrenal is highest, however, in the various organs (12), and oleic acid richly contained in olive oil (OLI) is reported to be best as a substrate of acyl-CoA: cholesterol acyltransferase in the liver (13), which practically exists in all tissues. The feeding of OLI to rats enhances the concentration of plasma total CHOL in the previous reports (14-16). It is considered that in rats loaded with stress, the feeding of OLI increases plasma CHOL level, and as a result the incorporation of CHOL into the adrenal is elevated, by which the secretion of adrenal corticosterone is enhanced. Few investigations, however, are involved in the relationships between stress loading and various oils. The present study was conducted to compare the effects of OLI on stress-induced changes with other oils

* To whom request for reprint should be addressed; 2787 34, Ohya, Shizuoka 422-8017, Japan. in experimentally stress-loaded rats, adopting the water immersion of rats individually restrained in cages (17), which is similar to a cold restraint normally regarded as a primary physical-stress $(5,7)$. The present stress load was divided into single and repetitive stresses, as the responses of a living body on these stresses were conjectured to be different between both ones (5).

\section{MATERIALS AND METHODS}

Materials. Casein, OLI, safflower oil (SAF), linseed oil (LIS), tripalmitin, and tristearin (Nacalai Tesque, Kyoto), sugar (Fuji Seito, Co. Ltd., Shimizu), and mineral and vitamin mixtures (AIN-76MA) (18) (Nihon Nosan Kogyo, Ltd., Yokohama) were purchased from respective companies. Corn starch $(\alpha)$ and corn oil were presented by Fuji Seifun, Ltd., and Honen Corporation (Shimizu), respectively.

Animals and diets. Male rats of the Wistar strain (Japan SLC, Hamamatsu), about $150 \mathrm{~g}$ and $6 \mathrm{wk}$ of age, were individually housed in suspended stainless-steel wire cages and fed in an air-conditioned room with a temperature of $23 \pm 1{ }^{\circ} \mathrm{C}$, a humidity of $50 \pm 3 \%$, and a $12 \mathrm{~h}$ light (06:00-18:00) and dark cycle. They had free access to food and water. The experimental plan was approved by the Experimental Animal Care Committee of the Faculty of Agriculture, Shizuoka University. The 
Table 1. Fatty acid composition of oils ${ }^{1}$ used.

\begin{tabular}{lrrrr}
\hline Fatty acid & PSC & \multicolumn{1}{c}{ OLI $\begin{array}{c}\text { SAF } \\
(w t \%)\end{array}$} \\
& \multicolumn{4}{c}{ LIS } \\
\hline $16: 0$ & 66.2 & 8.1 & 6.9 & 5.6 \\
$18: 0$ & 16.4 & 2.4 & 2.5 & 3.4 \\
$18: 1 n-9$ & 6.9 & 75.1 & 12.9 & 19.3 \\
$18: 3 n-6$ & 10.0 & 12.7 & 76.6 & 15.8 \\
$18: 3 n-3$ & 0.3 & 0.6 & 0.3 & 55.5 \\
Others & 0.2 & 1.1 & 0.8 & 0.4 \\
\hline
\end{tabular}

${ }^{1}$ PSC, the mixture of tripalmitin (64\%), tristearin (16\%), and corn oil (20\%); OLI, olive oil; SAF, safflower oil; LIS, linseed oil.

rats were fed a basal diet prepared on the dietary recommendations of the American Institute of Nutrition (18), except for the variation in dietary protein level, to accustom themselves to the surroundings for 4 to $5 \mathrm{~d}$. The basal diet contained (in weight \%) casein (25.0), mineral mixture (3.5), vitamin mixture (1.0), choline chloride (0.2), corn oil (10.0), sucrose (46.2), and $\alpha$-starch (14.1), which was added $0.1 \mathrm{~mL}$ of $20 \%$ BHT in alcohol per $100 \mathrm{~g}$ of the diet.

In the experiment (Exp.) 1 (single stress), rats having about $170 \mathrm{~g}$ of body weight were divided into four groups of 12 rats each, which received any of the test diets containing $20 \%$ of the mixture of tripalmitin, tristearin, and corn oil (PSC), OLI, SAF, and LIS (PSC, OLI, SAF, and LIS) for $10 \mathrm{~d}$. The PSC was semisynthesized by mixing $64 \%$ of tripalmitin, $16 \%$ of tristearin, and $20 \%$ of corn oil. The test diets were prepared by increasing the content of oils at the expense of sucrose and $\alpha$ starch in the basal diet, keeping the constant rate (46.2/14.1) between them. The fatty acid compositions of dietary oils are shown in Table 1. At the end of the feeding period, all groups were food-deprived for $5 \mathrm{~h}$, and half the rats of each group was once loaded with water immersion-restraint stress (single stress) for the latter $3 \mathrm{~h}$ in the term of food deprivation. Immediately after treatments, rats were killed by decapitation and blood samples were collected into polyethylene tubes with heparine, and the liver, spleen, thymus, and adrenal were removed and weighed. The plasma was obtained by centrifuging at $900 \times g$ for $10 \mathrm{~min}$. The stress was basically performed by the method of Takagi and Okabe (17). A stress cage divided into 10 compartments, each keeping a rat, was immersed in the water bath $\left(25^{\circ} \mathrm{C}\right)$ up to the rats' necks for $3 \mathrm{~h}$.

In Exp. 2 (repetitive stress), rats weighing about $170 \mathrm{~g}$ were divided into four groups of 12 rats each and fed the test diets presented in Exp. 1 for 7 d. Half the rats in each group was repetitively exposed to the water immersion-restraint stress (repetitive stress) every other day (a total of 4 times) for $2 \mathrm{~h}$ during the feeding period. At the end of feeding, all groups were deprived of food for $5 \mathrm{~h}$, and the stress groups were loaded with the final stress for the latter $2 \mathrm{~h}$ during the term of food deprivation. The other treatments were performed as described in Exp. 1.
All samples were stocked at $-20^{\circ} \mathrm{C}$ until analyzed.

Analytical procedure. The fatty acid compositions of oils used were determined by the previous report (16). The concentrations of total (T)-CHOL, triglyceride (TG), and urea in the plasma were determined by using enzymatic kits (Wako Pure Chemical Industries, Osaka). The levels of plasma corticosterone and lipid peroxide were estimated by a fluorescent method (19) and Yagi's method (20), respectively. The amounts of liver T-CHOL and TG were measured by the methods of Zak-Henley (21) and Fletcher (22), respectively, after the extraction of lipids with the mixture of chloroform-methanol $(2: 1, \mathrm{v} / \mathrm{v})(23)$ from the freeze-dried liver.

Statistical analysis. Data were presented as the mean \pm SE for 6 rats of each group and subjected to a one-way variance (ANOVA), followed by the inspection of all differences among means by using Duncan's multiple range test (24) at $p<0.05$. The correlation coefficient $(r)$ was determined by the method of least squares.

\section{RESULTS}

Body weight gain, food intake, and organ weight

The changes in final body weight, body weight gain, food intake, and liver weight of rats exposed to the single stress or not after the feeding of test diets for $10 \mathrm{~d}$ (Exp. 1) are shown in Table 2. The final body weights were scarcely different among the groups. The weight gains were higher in stress-unexposed (normal) SAF and LIS groups than in the other groups. The food intakes were not significantly different among dietary groups. The liver weights were hardly different among all groups except the normal LIS group, of which liver weights tended to be increased compared with the other groups. The weights of the spleen, thymus, and adrenal were unchanged among dietary groups, though the data were not shown.

The final body weights, weight gains, food intakes, and organ weights in rats loaded with the repetitive stress or not during the feed of test diets (Exp. 2) are presented in Table 2 . The final body weights were generally smaller in normal groups than in stressed ones. The difference in weight gain among normal groups was not observed except in the PSC group, in which weight gain was lower than in the other groups, but stress loading reduced the weight gains of all groups. The change in food intake was nearly in proportion to weight gain in the groups fed any of test diets. Liver weights were not different among the groups. The weights of the thymus and spleen were hardly changed by types of oil, but they were reduced by stress-loading. The adrenal weights were generally inclined to be increased by stress load in all groups except the PSC; especially the stress-exposed OLI group had the greatest adrenal weight.

T-CHOL, TG, urea, lipid peroxide, and corticosterone in the plasma and T-CHOL and TG in the liver

The concentrations of T-CHOL, TG, urea, lipid peroxide and corticosterone in the plasma, and T-CHOL and TG in the liver of rats loaded or not loaded with the single or repetitive stress (Exp. 1) are presented in Table 3 and Fig. 1. As shown in Table 3, the concentrations of 


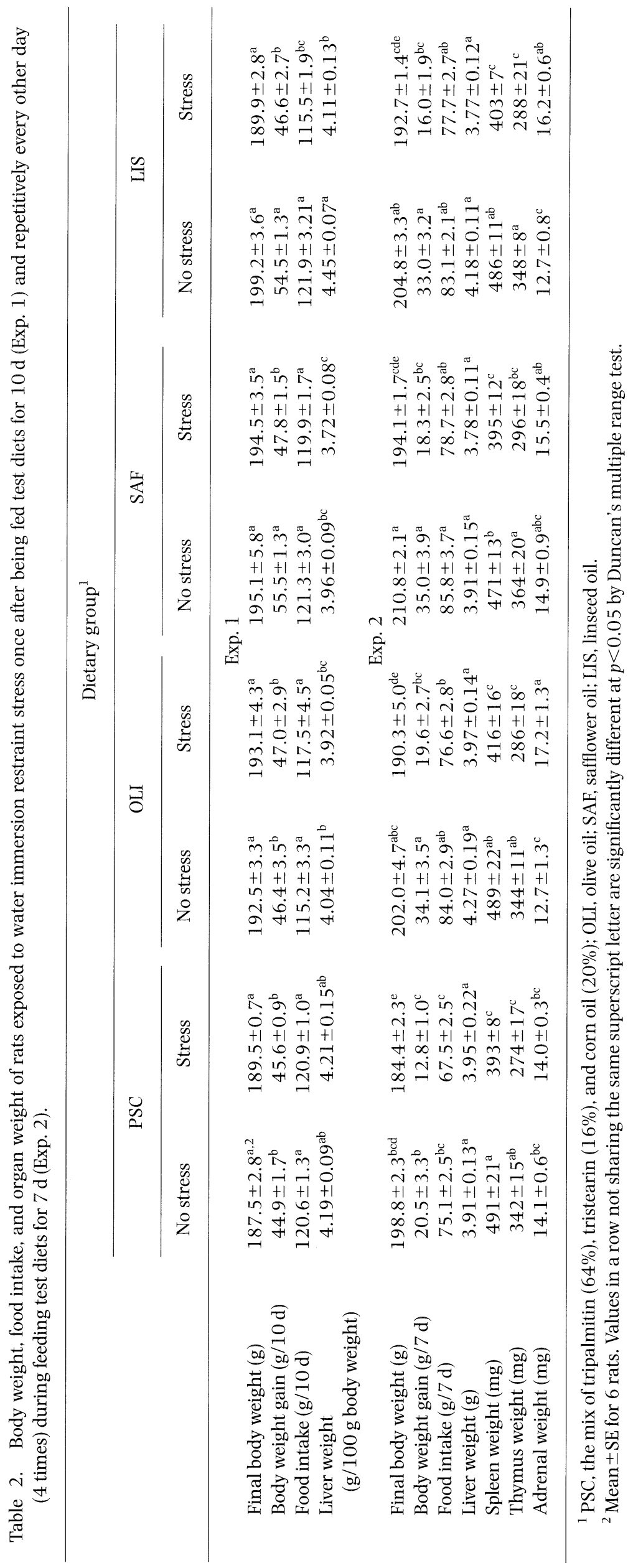




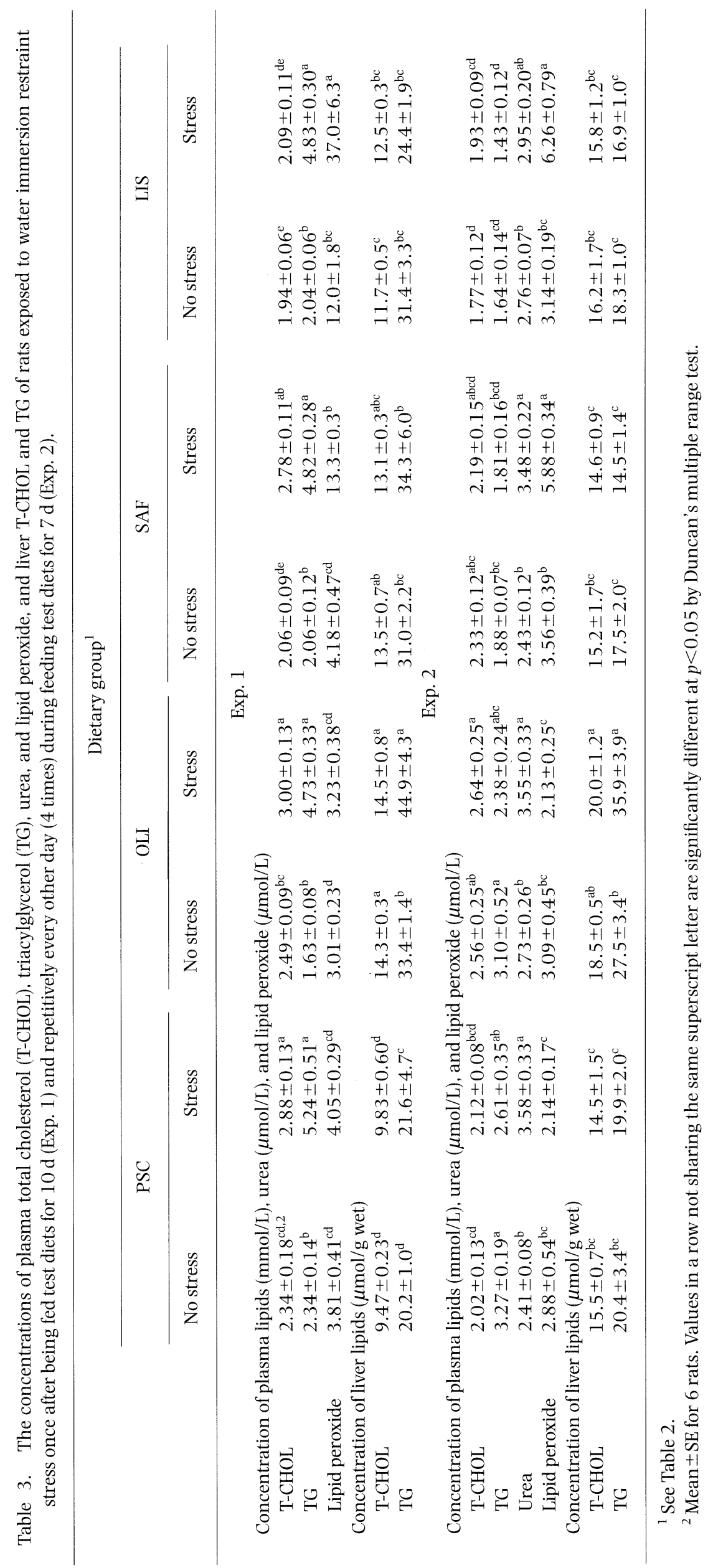




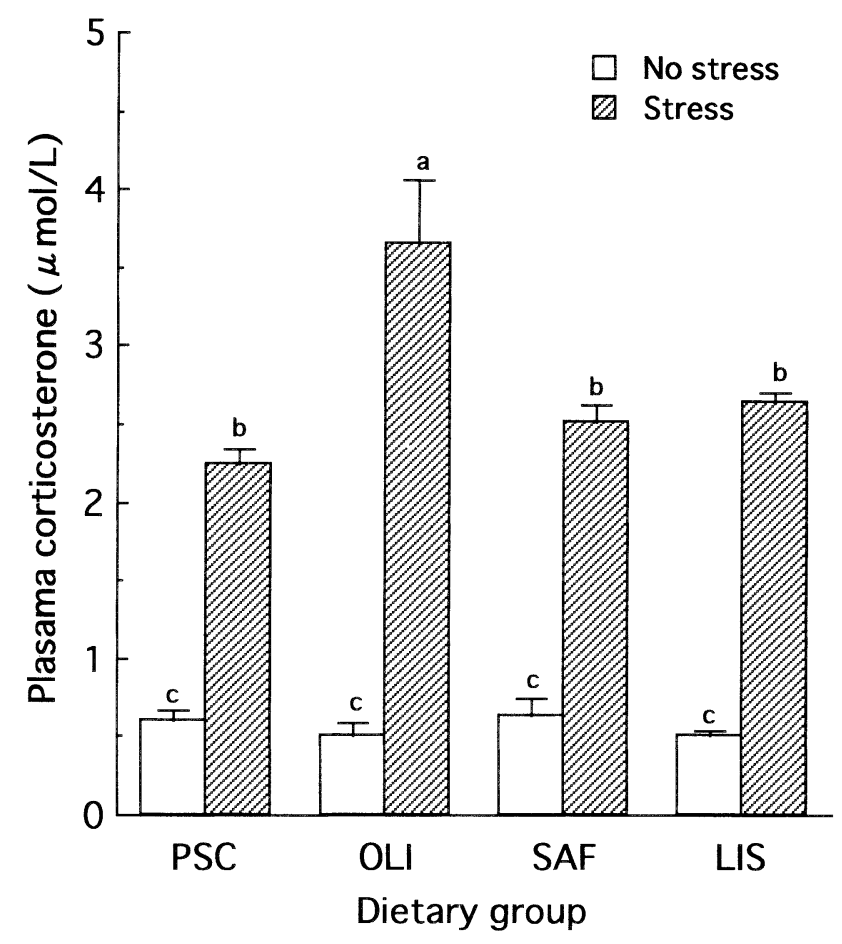

Fig. 1. Effect of dietary oils on plasma corticosterone concentrations of one-time water immersion restrained rats. Rats were once exposed to the water immersion restraint stress after being fed test diets for $7 \mathrm{~d}$. Vertical bars represent the SE of means for 6 rats. The bars with different superscript letters are significantly different at $p<0.05$ by Duncan's multiple range test. The abbreviations for dietary groups are as follows: PSC, the mixture of tripalmitin (64\%), tristearin (16\%), and corn oil (20\%); OLI, olive oil; SAF, safflower oil; LIS, linseed oil.

plasma T-CHOL were generally higher in normal PSC and OLI groups than in the normal SAF and LIS groups, and they were significantly increased by stress exposure in all groups except the LIS group. Plasma TG concentrations were hardly different among normal groups, but were enhanced by stress loading in each group, among which difference was scarcely observed. Plasma urea levels were unchanged by dietary oils, but they were extremely increased by stress in all groups, among which there was no difference in the urea levels. The concentrations of lipid peroxide were highest in the stress-loaded LIS group, next high in the stressed SAF and normal LIS groups, and lowest in the other groups. Plasma corticosterone levels (Fig. 1) were low in normal groups, among which there was no difference in the levels, but they were enhanced by stress load in each group. The rise of this hormone level by stress were especially greater in the OLI group than in the other groups.

The contents of liver T-CHOL and TG had an inclination to be higher in the OLI group than in the other groups with and without stress exposure.

The levels of T-CHOL, TG, urea, lipid peroxide, and corticosterone in the plasma and T-CHOL and TG in the liver of rats exposed or unexposed to the repetitive stress during feeding test diets (Exp. 2) are shown in Table 3

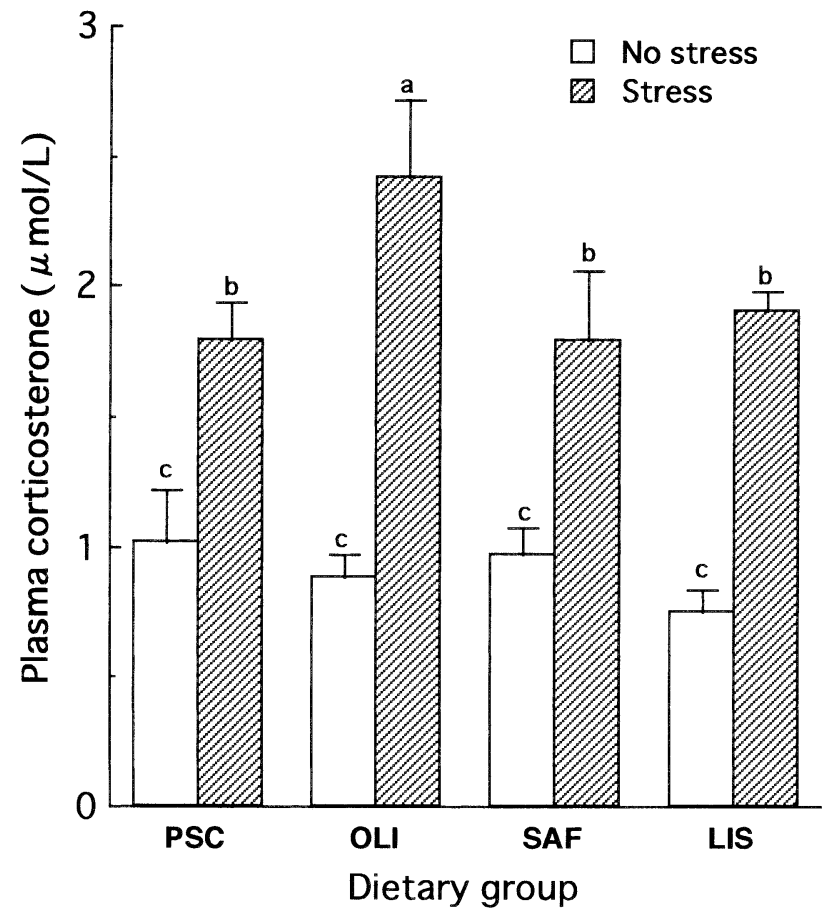

Fig. 2. Effect of dietary oils on plasma corticosterone concentrations of repetitively water immersion restrained rats. The rats were repetitively exposed to the water immersion restraint stress every other day (4 times) during the feeding of test diets for $7 \mathrm{~d}$. Vertical bars represent the SE of means for 6 rats. The bars with different superscript letters are significantly different at $p<0.05$ by Duncan's multiple range test. The abbreviations for dietary groups are shown in Fig. 1.

and Fig. 2. The concentrations of plasma T-CHOL were inclined to be highest with feeding of the OLI diet, followed in order by the PSC and SAF diets and lowest in the LIS diet. No difference was found in the concentrations between the load and nonload of stress. Plasma TG levels were generally higher in normal rats fed the PSC and OLI diets than in the other diets, but they were inclined to be reduced by stress exposure in both groups. Urea concentrations in the plasma were hardly different among each normal group, and they similarly increased with stress exposure in any group except LIS, in which the urea level was not increased by stress. The contents of plasma lipid peroxide were scarcely different among normal groups, but they extremely rose in the SAF and LIS groups under stress treatment. The livers generally contained the higher amounts of T-CHOL and TG in rats given the OLI diet than in rats fed the other diets under normal and stress load. No significant difference was noted in plasma corticosterone levels among normal groups, but stress loading extremely raised the levels in any of the groups, and enhancement in the levels was especially great in the stressed OLI group (Fig. 2).

Correlation between corticosterone and adrenal weight

As shown in Fig. 3, a positive correlation was found between plasma corticosterone concentrations and the adrenal weights $(r=0.87, p<0.05)$. 


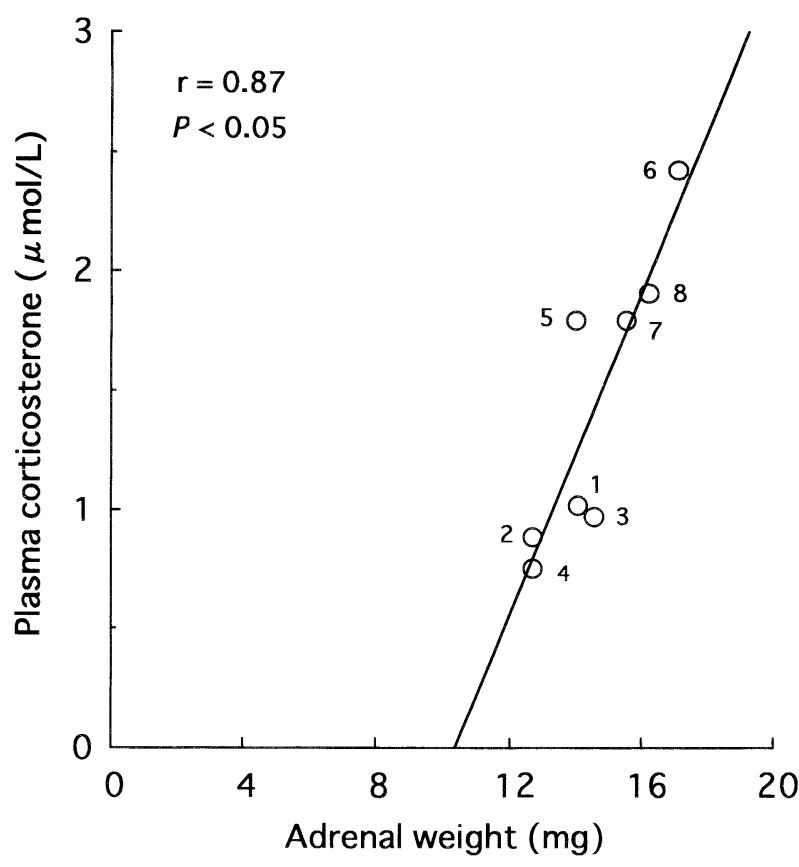

Fig. 3. Correlation between plasma corticosterone levels and adrenal weights in rats repetitively water immersion restrained or not. The symbols indicate the means for 6 rats. The numbers 1, 2, 3, and 4 represent the PSC, OLI, SAF, and LIS groups unloaded the stress. Those of 5, 6, 7, and 8 indicate the PSC, OLI, SAF, and LIS groups, which are loaded with the stress. The abbreviations for dietary groups are shown in Fig. 1.

\section{DISCUSSION}

The water immersion of restrained rats in this study is normally regarded as primary physical stress $(5,7)$, and similar stress has been used to induce ulcers in the digestive organs of rats $(17,25)$. The ulcers were not visibly found in Exps. 1 and 2, however. This observation suggests that the degree of the present stresses was relatively modest. In both experiments (Table 2), the body weight gains of normal rats fed the PSC diet tended to be reduced compared with those of normal rats fed the other diets, though there was no difference in food intake between the PSC group and the other groups. This decrease in weight gain would be due to stearic acid enriched in the PSC diet, since stearic acid is difficult to be absorbed in the intestine and as a result bring about a smaller energy consumption than in the other groups $(26,27)$. The greater weight gains of normal rats in Exp. 1 than in Exp. 2 would be because of the longer period of feeding test diets in Exp. 1 compared with Exp. 2. The repetitive stress load caused the decline in food intakes, which was similar to the results of the previous studies $(11,28)$. The weights of the spleen and thymus in Exp. 2 (Table 2) were decreased by loading with stress in rats given any of the diets, among which there was no difference. This result was identical to the earlier studies $(10,11)$. Adrenal weights (Table 2 ) were inversely increased by stress, which was also reported previously (10), but the degree of weight increase was different among dietary oils. This investigation showed that OLI gave the greatest increase in adre- nal weight in the repetitive stress-loaded rats. The increase in adrenal weight was also correlative to the enhancement of plasma corticosterone level by the repetitive stress (Fig. 3), but the increase in adrenal weight was not observed by the single stress (Exp. 1). This difference between single and repetitive stresses may show that the raise in plasma corticosterone concentration by stress loading was not essentially related to the increase in adrenal weight, but the repetitive stress with feeding of the diets might have a bad influence on the adrenal weight besides the adaptability to stress as described below, since the secretion of adrenal corticosterone was lower under the repetitive stress than under the single one (Figs. 1 and 2).

The T-CHOL and TG levels in the plasma and liver (Table 3) generally tended to be higher with the feeding of the OLI diet than with the feeding of the other diets in the normal and stressed rats of both experiments, which was similar to the earlier reports $(14-16,29)$. This result supports OLI having an effect to enhance the contents of T-CHOL and TG in the plasma and liver and suggests that these enhancements by the feeding of OLI are related to a further increase in the raised concentration of plasma corticosterone as again described below.

Plasma urea levels (Table 3) were raised by stress load regardless of types of dietary oils, which was identical with the results of the previous study (4). This rise of urea level would be due to protein catabolism accelerated by adrenal corticosterone for obtaining an energy source, since stress-exposed situations are thought to require plenty of energy.

The concentrations of plasma lipid peroxides (Table 3) were generally enhanced with feeding of the SAF diet to rats and further enhanced with the LIS diet compared with the PSC and OLI diets under stress exposure, which was similar to the previous papers $(6,7)$.

The stress-induced acceleration of corticosterone secretion from the adrenal (Figs. 1 and 2), which was reported previously $(4,5,7)$, was found to be greater with the feeding of the OLI diet than with the other diets in this investigation. The corticosterone secretion response raised by the stress is thought further to strengthen the defensive mechanism of a living body against various stresses (10). The further increase in plasma corticosterone by feeding OLI under the stress load is conjectured to be closely related to the elevated concentration of plasma and liver T-CHOL, since CHOL is the material for corticosterone synthesis, and oleic acid richly contained in OLI is reported to be the best substrate for liver acyl-CoA: cholesterol acyltransferase (ACAT) (13), which is considered to exist in many tissues and to be involved in the esterification of CHOL. A role of OLI as the source of the best substrate for ACAT may be more worthy of notice than the high concentration of plasma T-CHOL. Liver TG levels, however, were higher with the OLI diet than with the other diets under both stresses (Table 3). This result may also show the possibility that OLI accelerates a corticosterone secretion, since it is in general known that glucocorticoid (an analogue of corticosterone) stimulates the gluconeoge- 
nesis, which is involved in TG synthesis in the liver.

A few of the metabolic changes in this study, plasma urea concentrations, lipid peroxide contents in the plasma of rats fed the SAF and LIS diets, and the secretion of adrenal corticosterone, were greater with the single stress treatment than with the repetitive one, which resembled in the previous report (30). This difference might be attributed to the adaptability to stress load.

In conclusion, the OLI rich in oleic acid especially increases the adrenal weight in rats loaded with the repetitive water immersion restrained stress and enhances the secretion of adrenal corticosterone in rats exposed to the single and repetitive ones compared with the other oils. The mechanism explaining the actions of OLI was inferred to be related to the raised levels of T-CHOL and TG in the plasma and liver by the feeding of OLI

\section{REFERENCES}

1) Sigg EB, Keim KL. 1976. Plasma corticosterone following alterations of hypothalamic catechol amines in rats. Pharmacol Biochem Behav 4: 95-97.

2) Rowland NE, Antelman SM. 1976. Stress-induced hyperphagia and obesity in rats: A possible model for understanding human obesity. Science 191: 310-312.

3) Stein M, Schiavi RC, Camerino MS. 1976. Influence of brain and behavior on the immune system. Science 191: 435-440.

4) Beattie D. 1978. Physiological changes in rats exposed to cold/restraint stress. Life Sci 23: 2307-2314.

5) Quirce CM, Maickel RP. 1981. Alterations of biochemical parameters by acute and repetitive stress situation in mice. Psychoneuroendocrinology 6: 91-97.

6) Logani MK, Davies RE. 1981. Lipid oxidation: Biologic effects and antioxidants-A review. Lipids 15: 485495.

7) Capel ID, Dorell HM, Smallwood AE. 1983. The influence of cold-restrained stress on some components of the antioxidant defence system in the tissues of rats of various ages. J Toxicol Environ Health 11: 425-436.

8) Teshima H, Kubo C, Kihara H, Nagata S, Imada Y, Ago Y. 1983. Stress and immune diseases. Igaku no Ayumi (J Clin Exp Med) 125: 493-500.

9) Levine SP, Towell BL, Suarez AM, Knieriem LK, Harris MM, George JN. 1985. Platelet activation and secretion associated with emotional stress. Circulation 71: 1129-1134.

10) Selye H. 1965. Thymus and adrenals in the responses of the organism to injuries and intoxications. $\mathrm{Br} J \operatorname{Exp}$ Pathol 17: 234-235.

11) Alario P, Gamallo A, Beato MJ, Trancho G. 1987. Body weight gain, food intake and adrenal development in chronic noise stressed rats. Physiol Behav 40: 29-32.

12) Kovanen PT, Basu SK, Goldstein JL, Brown MS. 1979. Low density lipoprotein receptors in bovine adrenal cortex. II. Low density lipoprotein binding to membranes prepared from fresh tissue. Endocrinology 104: 610-616.

13) Sugano M, Imaizumi K. 1986. Esterification of cholesterol. In: Koresuteroru (Cholesterol) (Sugano M,
Imaizumi K, eds), p 136-137. Sankyo Press, Tokyo.

14) Lee JH, Fukumoto M, Ikeda I, Sugano M. 1991. Effect of oleic acid level under constant $n-6 / n-3$ and $\mathrm{P} / \mathrm{S}$ ratios of dietary fats on lipid metabolism in rats. Agric Biol Chem 55: 1793-1798.

15) Lu Y-F, Wu F-L. 1994. Effect of monounsaturated fatty acid under fixed $\mathrm{P} / \mathrm{S}$ and $n-6 / n-3$ ratios on lipid metabolism in rats. J Nutr Sci Vitaminol 40: 189-200.

16) Takeuchi $H$, Kato $T$, Ikegami $H$, Imai $H$, Takeuchi $H$. 1999. Regulation of plasma and liver total cholesterol levels by dietary oleic acid in rats fed a high-cholesterol diet. J Nutr Sci Vitaminol 45: 63-77.

17) Tagaki K, Okabe S. 1968. The effects of drugs on the production and recovery processes of the stress ulcer. Jpn J Pharmacol 18: 9-18.

18) The American Institute of Nutrition. 1977. Report of the American Institute of Nutrition Ad Hoc Committee on standards for nutritional studies. $J$ Nutr 107: 1340-1348.

19) Fujima S, Ikegawa S, Kishinami K. 1984. The measurement of cortisol by a fluorescent method. In: Seikagaku Bunsekiho (Biochemical Analysis Method) (Tamura Z, Yoshiki H, eds), p 424-425. Nankodo, Tokyo.

20) Yagi K. 1975. Microanalysis of plasma lipid peroxide. Igaku no Ayumi (J Clin Exp Med) 95: 93-94.

21) Kitamura M. 1968. The modified method of Zak-Henly. In: Clinical Chemical Analysis (Niwa M, Kitamura M, Saito M, eds), Vol 3, p 72-78. Tokyo Kagakudozin, Tokyo.

22) Fletcher MJ. 1968. A colorimetric method for estimating serum triglycerides. J Clin Chem Acta 22: 393-397.

23) Folch J, Dees M, Sloane-Stanley GH. 1957. A simple method for the isolation and purification of total lipids from animal tissues. J Biol Chem 226: 497-509.

24) Duncan DB. 1957. Multiple range tests for correlated and heteroscedastic means. Biometrics 13: 164-176.

25) Senay EC, Levine RJ. 1967. Synergism between cold and restraint for rapid production of stress ulcers in rats. Proc Soc Exp Biol Med 124: 1221-1223.

26) Mattson FH. 1959. The absorbability of stearic acid when fed as a simple or mixed triglyceride. J Nutr $\mathbf{6 9}$ : $338-342$.

27) Feldman EB, Russel BS, Schnare FH, Moretti-Rojas I, Miles BC, Doyle EA. 1979. Effects of diets of homogeneous saturated triglycerides on cholesterol balance in rats. J Nutr 109: 2237-2246.

28) Shibasaki T, Yamauchi N, Kato Y, Masuda A, Imaki T, Hotta M, Demura H, Oono H, Ling N, Shizume K. 1988. Involvement of corticosterone releasing factor in restraint stress-induced anorexia and reversion of the anorexia by somatostatin in the rat. Life Sci 14: 1103-1105.

29) Kobatake Y, Kuroda K, Jinnouchi H, Nishide E, Innami S. 1984. Differential effects of dietary eicosapentaenoic and docosahexaenoic acids on lowering of triglyceride and cholesterol levels in the serum of rats on hypercholesterolemic diet. J Nutr Sci Vitaminol 30: 357-372.

30) Lomakina LV. 1980. Effect of low temperature on lipid peroxidation and intensity of proteolysis in rat brain and liver tissues. Ukr Biokhim Zh 52: 305-308. 